\title{
具有同质多晶依赖性发射的非芳香性发光化合物
}

\author{
来悦颖赵子豪郑书源袁望章* \\ (上海交通大学化学化工学院 变革性分子前沿科学中心 上海市电气绝缘与热老化重点实验室 上海 200240)
}

\begin{abstract}
摘要 不含芳(杂)环、单键-重键交替单元等大共轭结构的纯有机发光材料引起了人们的广泛关注, 但其结构与发光性 质间的关系仍需进一步明确. 本文研究了氧杂酸䣶与氧杂酰亚胺两种非芳香性发光化合物. 通过在不同溶剂中培养单 晶, 得到了具有不同发光颜色及荧光-磷光双发射的同质多晶体, 发光效率最高达 $17.0 \%$. 这种高效发射可归因于含 $\pi$ 电子与 $\mathrm{n}$ 电子基团的簇聚产生的空间共轭及构象刚硬化. 尽管这些同质多晶体结构差异微小, 但少量溶剂分子的存在 导致簇聚体微环境改变, 调节了单线态与三线态发射比例, 从而产生了不同颜色的发光. 这些结果有助于人们进一步 了解非典型发光化合物构象与发光间的关系, 为其机理研究与分子设计提供了新的启示.
\end{abstract}

关键词 非芳香性发光化合物; 同质多晶; 室温磷光; 簇聚诱导发光; 空间共轭

\section{Polymorphism-Dependent Emission of Nonaromatic Luminophores}

\author{
Lai, Yueying Zhao, Zihao Zheng, Shuyuan Yuan, Wang Zhang*
}

(School of Chemistry and Chemical Engineering, Frontiers Science Center for Transformative Molecules, Shanghai Key Lab of Electrical Insulation and Thermal Aging, Shanghai Jiao Tong University, Shanghai 200240, China)

\begin{abstract}
Recently, nonconventional luminophores have received increasing attention, owing to their fundamental importance, advantages in outstanding biocompatibility, easy preparation, environmental friendliness and potential applications in sensing, imaging, encryption, etc. In order to provide more information about relationship among molecular conformation, molecular packing and emission, and moreover, to guide the design of nonconventional luminophores, molecules with definite structures and explicit molecular packing are highly desired. In this contribution, we report two nonconventional luminophores, namely F-MA and F-MI, consisting of carbonyls $(\mathrm{C}=\mathrm{O})$, electron-rich heteroatoms $(\mathrm{O} / \mathrm{N})$, and unsaturated $\mathrm{C}=\mathrm{C}$ subgroups. They are nonluminous in dilute solutions while being emissive in concentrated ones. Furthermore, through crystallization in different solvents, polymorphs of both compounds with various emission colors along with distinct room temperature phosphorescence (RTP) are successfully obtained. Under $312 \mathrm{~nm}$ UV irradiation, three polymorphs of F-MA emit bluish-violet, blue and white lights, accompanying photoluminescence (PL) and RTP quantum efficiencies $\left(\Phi_{\mathrm{c}} / \Phi_{\mathrm{p}}\right)$ of $10.6 \% / 1.8 \%, 9.4 \% / 2.1 \%$ and $2.9 \% / 1.7 \%$, respectively. To acquire more efficient emission, hydrogen bonds are introduced via amidation of F-MA, leading to the target compound F-MI with strikingly improved PL performance. Notably, F-MI is also polymorphic, whose $\Phi_{\mathrm{c}}$ and $\Phi_{\mathrm{p}}$ are of up to $17.0 \%$ and $4.8 \%$, respectively. Meanwhile, the RTP lifetimes of F-MI polymorphs are significantly prolonged by 10- to 56-fold, as compared with their corresponding F-MA counterparts. The above PL properties can well be rationalized by the clustering-triggered emission (CTE) mechanism, namely through-space electronic delocalization of $\pi$ and $n$ electrons among different molecules in concentrated solutions or crystals alongside with sufficiently rigidified conformations is accountable for the emission, which is also verified by single crystal analysis and theoretical calculation. Besides, the noticeable RTP emission should be ascribed to the presence of $\mathrm{C}=\mathrm{O}$ and heteroatoms and the clustering of such subgroups, which are ready to enhance spin-orbit coupling (SOC) and subsequent intersystem crossing transitions with effective through-space conjugation. Surprisingly, subtle changes caused by trace solvents in molecular conformations and packing modes significantly impact on intra/intermolecular interactions, which alter the relative intensity of singlet (fluorescence) and triplet (RTP) emissions, thus resulting in polymorphism-dependent emission colors. For these unorthodox luminescent molecules, their PL properties of polymorphs will deepen the understanding of the relationship between subtle structure variation and emission, thus enlightening further luminescent mechanism understanding and future rational design of novel nonconventional luminophores.
\end{abstract}

Keywords nonaromatic luminophores; polymorphism; room temperature phosphorescence; clustering-triggered emission; through-space conjugation

*E-mail: wzhyuan@sjtu.edu.cn

Received August 15, 2020; published September 16, 2020.

Supporting information for this article is available free of charge via the Internet at http://sioc-journal.cn.

Project supported by the National Natural Science Foundation of China (No. 51822303) and the Natural Science Foundation of Shanghai (No. 20ZR1429400). 国家自然科学基金(No. 51822303)和上海市自然科学基金(No. 20ZR1429400)资助项目. 


\section{1 引言}

传统有机发光化合物通常含有芳(杂)环、单键-重键 交替单元等显著共轭结构 ${ }^{[1]}$, 近年来, 不含此类结构的 非典型发光化合物由于基础研究的重要意义及制备简 单、来源广泛、生物相容性良好、应用前景广阔等优点 而受到广泛关注 ${ }^{[2]}$. 这些化合物通常含有富电子单元, 如含孤对电子 $(n)$ 的 $N 、 O 、 S 、 P$ 等杂原子 ${ }^{[2-3]}$ 及含 $\pi$ 电 子的重键结构 $(\mathrm{C}=\mathrm{C}, \mathrm{C}=\mathrm{O}, \mathrm{CN} \text { 等 })^{[4]}$. 目前, 人们已在 大量非芳香性体系中观测到了此类发光现象, 包括合成 化合物体系、糖类等天然产物、生物分子及超分子体系, 如: 聚酰胺胺 $(\mathrm{PAMAM})^{[5]}$ 、聚丙烯腈 $(\mathrm{PAN})^{[6]}$ 、非芳香 性氨基酸 ${ }^{[7]}$ 、蛋白质 ${ }^{[8]}$ 等. 除苂光发射外, 这些化合物通 常还表现出显著的磷光发射, 甚至是室温磷光(RTP)或 超长寿命室温磷光(p-RTP $)^{[3 \mathrm{a}, 4 \mathrm{a}-\mathrm{-c} c]}$. 这不仅对研究体系发 光机理、开发新型发光材料具有重要意义, 也为开发其 应用奠定了基础. 但对体系构效关系的理解, 特别是发 光性质的调控, 仍缺乏认识, 亟待进一步深入探索. 同 时, 前期许多体系为聚合物 ${ }^{[5-9]}$, 其聚集结构的不确定性 使得对发光机制的理解充满挑战. 针对不同体系, 人们 提出了氧化 ${ }^{[10]}$ 、羰基聚集 ${ }^{[11]}$ 、蛋白中氢键形成及肽键电 子离域 ${ }^{[12]}$ 、质子转移 ${ }^{[13]}$ 、交联增强发光 ${ }^{[2,14]}$ 、富电子基 团簇聚诱导发光 $(\mathrm{CTE})^{[2 \mathrm{a}, 6]}$ 等机理, 但目前仍未达成共

(a)

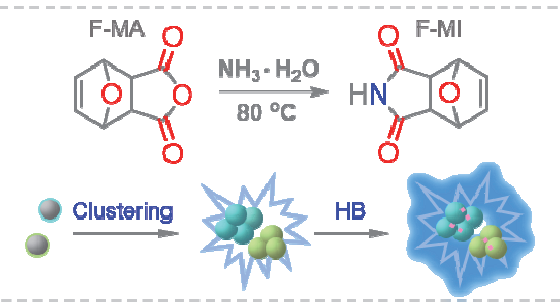

识. 深入理解发光机理并指导非典型发光化合物的设 计，构筑晶体结构明确的分子化合物并阐明其构效关系 显得无为关键 ${ }^{[3 a, 3 b, 4 a-4 c]}$.

晶体中分子构象和堆积模式的变化会影响分子内 和分子间相互作用, 从而形成同质多晶 ${ }^{[15-16]}$. 通过同质 多晶来调节化合物的发光也常见诸报道 ${ }^{[16]}$. 然而, 这些 化合物通常是具有典型共轭结构的传统发光分子 ${ }^{[17]}$, 非典型发光化合物的同质多晶发光鲜有报道. 在研究过 程中，我们发现氧杂酸䣶(F-MA)及其氨化产物 F-MI(图 $1 \mathrm{a})$ 在不同条件下培养单晶，能得到量子效率 $\left(\Phi_{\mathrm{c}}\right)$ 不同的 单晶. 特别地, 晶体均呈现出荧光 p-RTP 双发射. 氨化 后氢键的引入限制了分子运动, 使构象进一步刚硬化, 从而使 F-MI 晶体的效率 $\left(\Phi_{\mathrm{c}}\right)$ 及 p-RTP 寿命 $\left(<\tau>_{\mathrm{p}}\right)$ 较 F-MA 晶体高(图 1b). 因此, 同质多晶体及杂原子的改 变可有效地调节非典型发光化合物的 PL 性质. 值得注 意的是, F-MA, F-MI 各自同质多晶体分子构象差异微 小, 但光物理性质呈现显著差异, 这是由晶体中残存的 微量溶剂分子引起的. 溶剂导致晶体构象的微小变化, 进而调节了单线态与三线态的发射比率，产生具有不同 发光颜色的晶体. 对于非典型发光化合物，其同质多晶 现象将深化人们对构象变化与发光性质之间关系的了 解, 从而为其机理研究与分子设计提供新的启示.

(b)

\begin{tabular}{cccc}
\hline Sample & $\Phi / \%$ & $\Phi_{\mathrm{p}} / \%$ & \langle\rangle$_{\mathrm{p}} / \mathrm{ms}$ \\
\hline MA-A & 10.6 & 1.8 & 1.4 \\
MA-B & 9.4 & 2.1 & 1.1 \\
MA-C & 2.9 & 1.7 & 5.8 \\
MI-A & 17.0 & 4.3 & 63.2 \\
MI-B & 16.2 & 4.8 & 61.6 \\
MI-C & 6.8 & 1.8 & 58.0 \\
\hline
\end{tabular}

(c)

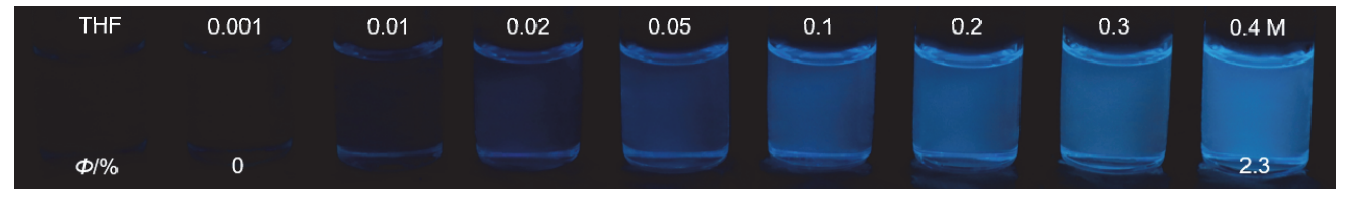

\section{(d)}

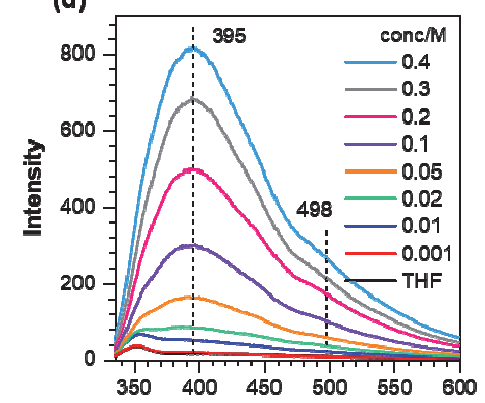

(e)

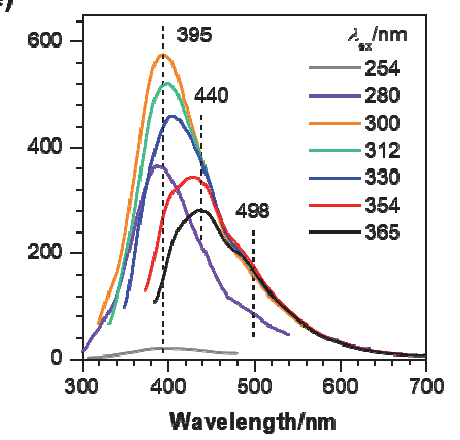

(f)

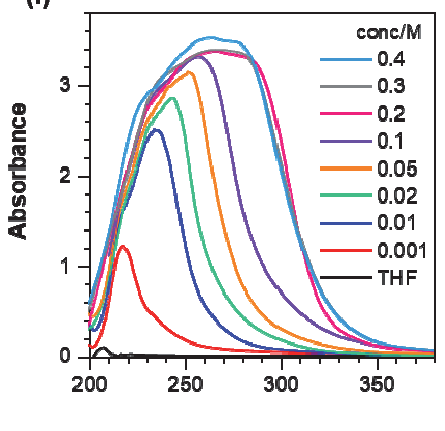

图 1 (a)F-MA 和 F-MI 的化学结构、发光机理示意图及(b)其晶体主要光物理参数; 不同浓度 F-MA/THF 溶液在 $312 \mathrm{~nm}$ 紫外灯辐照下的(c)照片 与(d)发射谱; (e) $0.4 \mathrm{~mol} / \mathrm{L}$ F-MA/THF 溶液在不同 $\lambda_{\mathrm{ex}}$ 下的发射谱; (f)不同浓度 F-MA/THF 溶液的吸收光谱

Figure 1 (a) Chemical structures, schematic diagram of the emission mechanism, and (b) photophysical parameters of the crystals for F-MA and F-MI. (c) Photographs and (d) emission spectra of F-MA/THF solutions at different concentrations with $312 \mathrm{~nm}$ UV irradiation. (e) Emission spectra of 0.4 mol/L F-MA/THF solutions at different $\lambda_{\text {ex }}$. (f) Absorption spectra of F-MA/THF solutions with different concentrations 


\section{2 结果与讨论}

\subsection{F-MA 的光物理性质}

两种化合物均通过重结晶过程纯化, 并通过核磁谱 (图 S1, S2)和单晶数据(Table S1)进一步确认了其结构. 室温下, 不同浓度的 F-MA/四氢呋喃(THF)溶液在 312 $\mathrm{nm}$ 紫外灯下的照片如图 1c 所示, 当其浓度小于 $10^{-3}$ $\mathrm{mol} / \mathrm{L}$ 时, F-MA 几乎不发光(量子效率 $\Phi_{\mathrm{s}} \approx 0$ ); 随着溶 液浓度逐渐增加, 其蓝色发光也逐渐增强. 当溶液浓度 达 $0.4 \mathrm{~mol} / \mathrm{L}$ 时, 其发光强度最高, $\Phi_{\mathrm{s}}$ 达到 $2.3 \%$. 相应发 射谱如图 1d 所示, 位于 $330 \mathrm{~nm}$ 的峰为 THF 溶剂峰, 而 $395 \mathrm{~nm}$ 的主峰和 $498 \mathrm{~nm}$ 的肩峰可归属为 F-MA 分子在 溶液中逐渐形成聚集体后的发射. $0.4 \mathrm{~mol} / \mathrm{L}$ F-MA 的浓 溶液还具有明显的激发波长 $\left(\lambda_{\mathrm{ex}}\right)$ 依赖性, 其发射主峰位 随着 $\lambda_{\mathrm{ex}}$ 的增加而红移, 荧光主峰位从 $395 \mathrm{~nm}$ 红移至 $440 \mathrm{~nm}$, 并伴随 $498 \mathrm{~nm}$ 处肩峰的出现(图 1e). 相应地, 如图 S3 所示, $0.4 \mathrm{~mol} / \mathrm{L} \mathrm{F-MA/THF}$ 溶液在 395, 440 和 $498 \mathrm{~nm}$ 处的发射峰, 其最佳激发峰位分别为 303,330 和 $352 \mathrm{~nm}$, 这说明不同发射峰位是由于不同的发射种存在 导致的，也证明了激发波长依赖性的发射本质上来自于 不同的簇聚发射种 ${ }^{[2 b, 18]}$. 此外, 紫外吸收曲线边缘随着 浓度的增加不断向长波方向扩展, 且吸收光谱中主峰位 的红移也表明了共轭程度增大的新聚集体的形成(图 $1 \mathrm{f})^{[19]}$. 同时, 动态光散射(DLS)数据进一步说明了聚集
体的形成. 如图 S4 所示，随着浓度的增加，聚集体的粒 径从 $64.8 \mathrm{~nm}(0.01 \mathrm{~mol} / \mathrm{L})$ 逐渐增加到 $154.7 \mathrm{~nm}(0.4$ $\mathrm{mol} / \mathrm{L})$. 同时, 由于聚集, 分子的振动转动在一定程度 上受限，因此发光增强.

浓度增强发光及浓溶液激发波长依赖性在非典型 发光化合物中比较常见, 可用 CTE 机理合理解 释 ${ }^{[2 \mathrm{a}, 2 \mathrm{~b}, 6,18-20]}$. 在稀溶液中, F-MA 处于单分子分散状态, 非典型生色团无法形成分子间族聚，因此有效共轭较 小，无法被有效激发; 同时稀溶液中分子内运动活跃， 因此也不具备产生有效发射的条件. 浓溶液中, 分子相 互靠近，非典型生色团产生族聚，形成族发射中心. 族 生色团内电子云彼此重叠, 形成了较大的空间共轭和有 效的电子离域, 使单线态、三线态能级较单分子降低, 且构象刚硬化程度增加. 同时，分子间其它相互作用也 有利于构象刚硬化，从而能共同抑制非辐射跃迁过程， 因而能产生可见光发射. $77 \mathrm{~K}$ 下, 浓度小于 $10^{-3} \mathrm{~mol} / \mathrm{L}$ 的稀溶液依然无法产生可见光发射, 而室温发光溶液在 $77 \mathrm{~K}$ 时发光进一步增强(图 S5). 这些结果说明构象刚硬 化并非发光的起因, 而只是有效促进发光的因素, 非典 型生色团间的簇聚才是发光的根本原因. 低温浓溶液停 止紫外激发后能观察到余辉, 其颜色具有明显的 $\lambda_{\mathrm{ex}}$ 依 赖性，进一步证明了发光来自溶液中不同的簇聚种以及 CTE 机理的合理性. (a)

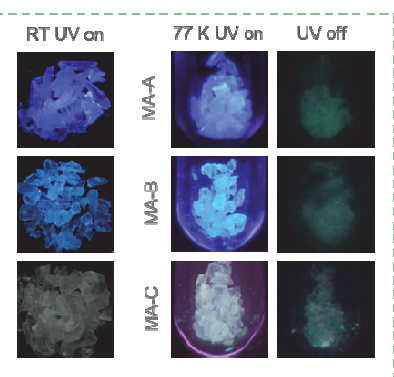

(d)

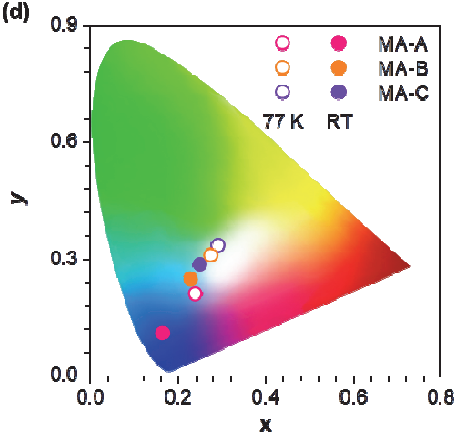

(b)

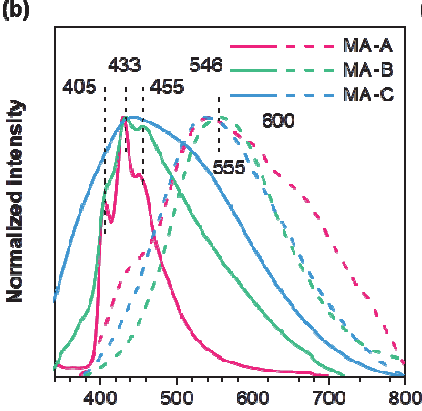

(e)

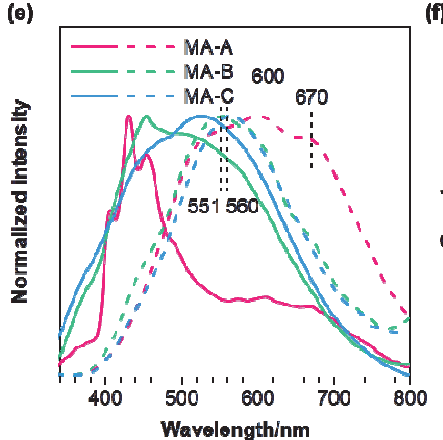

(c)

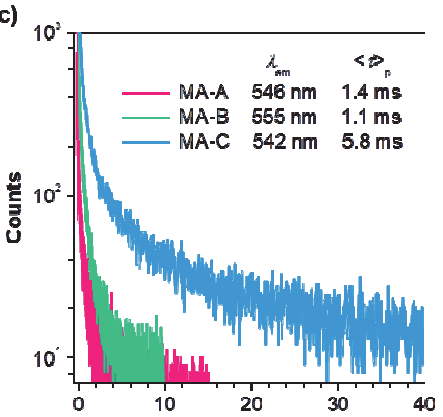

(f)

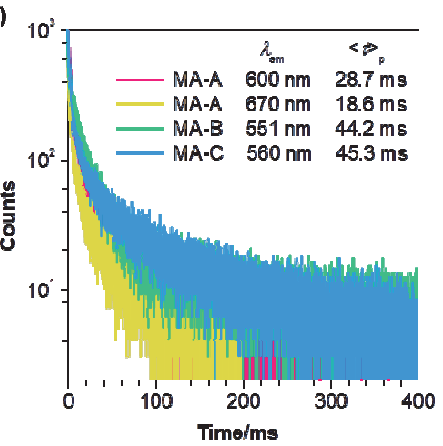

图 2 F-MA 同质多晶在(a)室温及 $77 \mathrm{~K}$ 时 $312 \mathrm{~nm}$ 紫外线照射前后的照片, (b)即时(实线, $t_{\mathrm{d}}=0 \mathrm{~ms}$ )与延迟(虚线, $t_{\mathrm{d}}=0.1 \mathrm{~ms}$ )发射光谱及(c)在 546 , 555 和 $542 \mathrm{~nm}$ 的发射衰减曲线; F-MA 的三种同质多晶在(d)室温及 $77 \mathrm{~K}$ 下的色坐标, (e) $77 \mathrm{~K}$ 下的发射光谱 $\left(\lambda_{\mathrm{ex}}=312 \mathrm{~nm}\right.$ )及(f) $600,670,551$ 和 560 $\mathrm{nm}$ 处的发射衰减曲线

Figure 2 (a) Photographs under and after $312 \mathrm{~nm}$ UV irradiation at room temperature and $77 \mathrm{~K}$, (b) prompt (solid line) and delayed (dash line) emission spectra and (c) emission decay profiles monitored at 546, 555 and $542 \mathrm{~nm}$ at room temperature of F-MA polymorphs. (d) The chromaticity coordinate at $77 \mathrm{~K}$ and room temperature, (e) emission spectra $\left(\lambda_{\mathrm{ex}}=312 \mathrm{~nm}\right)$ and (d) decay profiles monitored at $600,670,551$ and $560 \mathrm{~nm}$ of F-MA polymorphs at 77 $\mathrm{K}$ 
除荧光外, RTP 在非典型发光化合物中也常存在 ${ }^{[2 b]}$. 通过结晶来抑制分子运动并隔离猝灭源是实现纯有机 RTP 的重要手段 ${ }^{[21]}$, 因此我们进一步研究了 F-MA 晶体 的光物理性质. 通过在不同条件下培养单晶, 我们得到 了三种发光颜色不同的晶体. 在 $312 \mathrm{~nm}$ 紫外灯下, 从二 氯甲烷(DCM)中重结晶得到的 MA-A 晶体发出蓝紫色 光; 从乙酸乙酯(EA)中得到的 MA-B 晶体发蓝光; 从 DCM 中挥发得到的 MA-C 晶体发出较暗的淡白光(图 2a). 三种晶体在 405/433/455 nm 附近均出现发射峰位. 不同的是, 它们位于 $540 \mathrm{~nm}$ 处的肩峰比例由 MA-A 至 MA-C 逐渐增加(图 $2 \mathrm{~b}$ ), 且 $\Phi_{\mathrm{c}}$ 逐渐下降, 分别为 $10.6 \%, 9.4 \%$ 和 $2.9 \%$. 室温下, 停止紫外光激发后, 虽肉 眼无法观察到长余辉, 但延迟发射显示三种晶体均具有 RTP 发射. 其发射谱主峰较为相似, 均位于 $550 \mathrm{~nm}$ 附 近, 对应的磷光效率分别为 $1.8 \%, 2.1 \%$ 和 $1.7 \%$, 在 546, 555 和 $542 \mathrm{~nm}$ 处的 $\left\langle\tau>_{\mathrm{p}}\right.$ 分别为 1.4, 1.1 和 $5.8 \mathrm{~ms}$ (图 $1 \mathrm{~b}$, 图 2c). 特别地, 在 MA-A 中还观察到位于 600 和 $670 \mathrm{~nm}$ 处的两个小肩峰, 这可能是由不同的发射中心产生的.

$77 \mathrm{~K}$ 时, 三种晶体显示出与室温相近的发光颜色, 其强度明显增加. 此外, 在撤除 $312 \mathrm{~nm}$ 光激发后, 肉眼 可捕捉到长达 $7 \mathrm{~s}$ 的绿色磷光(图 2a). 其低温发射谱的 荧光主峰几乎没有变化, 但磷光所占比例显著增加(图 2e). MA-A 晶体磷光主峰位于 $600 \mathrm{~nm}, 546 \mathrm{~nm}$ 处则变为 肩峰, 且 $670 \mathrm{~nm}$ 处的峰更加明显; MA-B 和 MA-C 晶体 的主峰位没有显著变化, 但在 600 及 $670 \mathrm{~nm}$ 处出现了微 弱的肩峰, 这说明了三种同质多晶体具有相似的发射 种, 多个峰位的形成是由晶体中不同的簇聚生色团导致 的. 与室温相比, 低温磷光比例的增加导致三种晶体的 色坐标更接近白光区域(图 2d). 低温下, 分子构象刚性 显著增加, 限制了分子运动并进一步抑制了猝灭作用, 因此发光强度增强, 磷光比例上升, 同时 $\left\langle\tau>_{\mathrm{p}}\right.$ 也随之分 别延长至 28.7, 44.2 及 $45.3 \mathrm{~ms}$ (图 2f). 此外, MA-A 晶体 在 $670 \mathrm{~nm}$ 处的寿命为 $18.6 \mathrm{~ms}$, 磷光峰表现出明显的激 发波长依赖性(图 S6), 进一步证实了晶体中不同簇聚发 射种的存在.

\subsection{F-MI 的光物理性质}

为调节化合物发光, 并进一步稳定三线态激子, 实 现 p-RTP 发射, 我们通过氨化 F-MA 得到了 F-MI(图 1a). 同样, 通过在氨水、EA 和 DCM 中培养单晶, 我们 得到了 F-MI 的同质多晶体 MI-A, MI-B 及 MI-C. 在 $312 \mathrm{~nm}$ 紫外灯照射下, MI-A, MI-B 及 MI-C 分别发出蓝 光、黄白光和蓝紫光, 停止激发后, 均具有持续约 $1.5 \mathrm{~s}$ 的绿色余辉(图 3a), 其可归属为 p-RTP 发射. MI-A 发射 光谱在 $364,411,434,462 \mathrm{~nm}$ 处出现多个发射峰, 不同 $\lambda_{\mathrm{ex}}$ 下峰位、相对强弱及寿命不同(图 S7), 说明其归属于 多重发射中心. 与 MI-A 晶体不同, MI-B 和 MI-C 则表 现出整个可见光区域内的宽峰, 且在 MA 峰位附近也有 类似的峰或肩峰出现(图 3b), 说明两种晶体可能形成了
相似的发射种，其发射强度的比例不同导致发光颜色的 改变. 延迟 $0.1 \mathrm{~ms}$ 后, MI-A, MI-B 和 MI-C 分别在 517, 524 和 $529 \mathrm{~nm}$ 出现 p-RTP 峰, 其 $<\tau>_{\mathrm{p}} / \Phi_{\mathrm{p}}$ 分别为 63.2 $\mathrm{ms} / 4.3 \%, 61.6 \mathrm{~ms} / 4.8 \%$ 和 $58.0 \mathrm{~ms} / 1.8 \%$ (图 3c), 较 F-MA 明显增加. 这一结果表明氢键的引入促进了生色团簇 聚、增强了分子间相互作用, 从而有利于发光和 p-RTP 发射. F-MI 在低温下光物理性质的变化趋势与 F-MA 类 似: 晶体的发射强度及磷光比例较室温时增加, $\langle\tau\rangle_{\mathrm{p}}$ 分 别延长至 110.2, 94.6 和 $159.3 \mathrm{~ms}$ (图 S8). 此外, F-MI 溶 液同样表现出浓度增强发光现象(图 S9), 随浓度增加, 聚集体的粒径增加(图 S10), 紫外吸收峰红移, 长波处 相对强度也随之增加. 其浓溶液在室温及 $77 \mathrm{~K}$ 均呈现 出明显的 $\lambda_{\mathrm{ex}}$ 依赖性发射(图 S9). 这些现象进一步说明 了在浓度增加过程中不同簇聚发射种的形成，揭示了 CTE 机理的合理性.
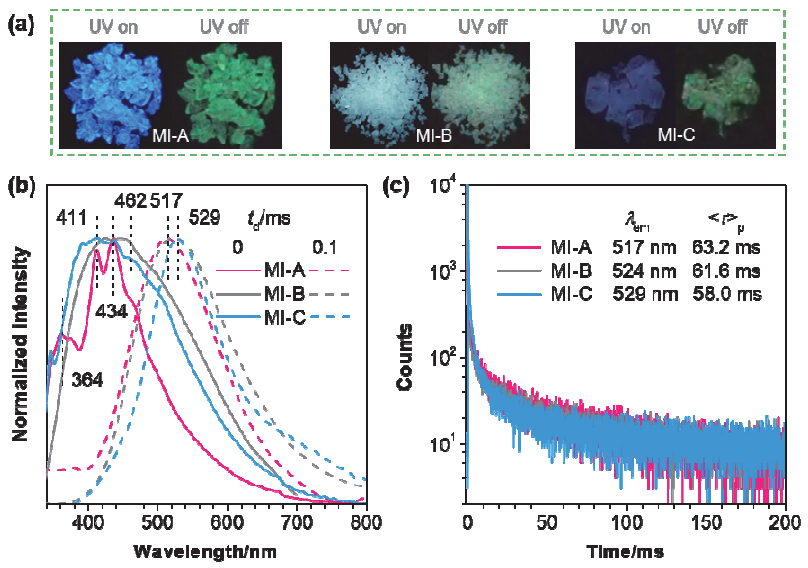

图 3 F-MI 同质多晶(a)在 $312 \mathrm{~nm}$ 紫外灯辐照及关闭辐照后的照片、 (b)即时与延迟 $\left(t_{\mathrm{d}}=0.1 \mathrm{~ms}\right)$ 发射光谱 $\left(\lambda_{\mathrm{ex}}=312 \mathrm{~nm}\right)$ 及(c)在 517,524 和 $529 \mathrm{~nm}$ 处的 $\mathrm{p}$-RTP 衰减曲线

Figure 3 (a) Photographs taken under and after ceasing the $312 \mathrm{~nm} \mathrm{UV}$ irradiation, (b) prompt and delayed $\left(t_{\mathrm{d}}=0.1 \mathrm{~ms}\right)$ emission spectra $\left(\lambda_{\mathrm{ex}}=\right.$ $312 \mathrm{~nm}$ ), and (c) p-RTP decay profiles monitored at 517, 524 and $529 \mathrm{~nm}$ at room temperature of $\mathbf{F}$-MI polymorphs

\section{3 单晶结构解析与发光机理}

需要指出的是, 上述发光是化合物的本征特性，而 非来源于杂质，这可从不同商业来源(Aladdin, TCI)及实 验室合成的 F-MA 晶体呈现类似的光物理现象得到证 明(图 S11). 为进一步探究分子结构与发光性质间的关 系，获得更多的发光机理信息，我们解析了化合物同质 多晶体的单晶结构 ${ }^{[22]}$. 以 $\mathbf{M A - A}$ 为例, 其分子周围存在 着众多分子间相互作用，包括 $\mathrm{C}-\mathrm{O} \cdots \mathrm{H}-\mathrm{C}(2.583$, $2.678 \AA), \mathrm{C}=\mathrm{O} \cdots \mathrm{H}-\mathrm{C}(2.500,2.507 \AA)$ 以及 $\mathrm{C} \cdots \mathrm{O}$ $(3.104,3.203 \AA$ ) 等短程接触(图 4a, S12), 这些作用力可 有效限制分子运动 ${ }^{[23]}$, 获得较为刚性的构象. 此外, 分 子内 $\mathrm{C}=\mathrm{O}$ 与桥键上 $\mathrm{O}$ 原子间及 $\mathrm{C}=\mathrm{C}$ 彼此距离较短, 从而产生分子内空间共轭, 扩展了电子离域程度(图 4a). 而分子间也存在诸如 $\mathrm{C}=\mathrm{O} \cdots \mathrm{O}-\mathrm{C}(3.498 \AA)$ 及 
$\mathrm{C}=\mathrm{C} \cdots \mathrm{O}-\mathrm{C}(3.203 \AA)$ 等相互作用，从而产生分子间空 间共轭，减小了基态与激发态间的能隙，从而使分子在 聚集态具有可见光发射能力 ${ }^{[24]}$. 特别地, MA-A, MA-B, MA-C 虽具有近乎相同的晶胞参数、密度与排列方式(图 $\mathrm{S} 12$, 表 $\mathrm{S} 1$ ), 但曾有文献报道, 键长 $0.008 \AA^{[16 b]}$ 或键角 $0.1 \sim 0.3^{\circ[17 b]}$ 的改变, 也会引起发光性质的巨大差异, 因 此分子内的一些细微差别不容忽视. 如图 S7 所示, 由 MA-A 到 MA-C, 以 $\mathrm{O}_{1}, \mathrm{O}_{2}$ 原子为中心, 分子内两侧 $\mathrm{C}-\mathrm{O}$ 键长差值增加, 分子构象的不对称性也增加. MA-C 相对扭曲的构象使分子内张力较大, 较强的分子 振动耗散了激子能量并导致较低的发光效率. 其次, 以 $\mathrm{O}_{1}, \mathrm{O}_{2}$ 为中心的 $\mathrm{C}-\mathrm{O}-\mathrm{C}$ 之间的夹角 $(\angle \mathrm{COC})$ 略有不同 (表 S2, 图 S12). MA-A 中夹角分别为 $110.43^{\circ}$ 和 $96.20^{\circ}$, 小于 $\mathrm{MA-B}\left(110.52^{\circ}, 96.34^{\circ}\right)$ 和 $\mathrm{MA-C}\left(110.68^{\circ}, 96.37^{\circ}\right)$ 的 角度. $\angle \mathrm{COC}$ 的差异会影响电子云的空间分布和簇生
色团的微环境，进而调节单线态和三线态的发射比例， 导致由 MA-A 到 MA-C 发光颜色改变.

对 MI-A, MI-B 和 MI-C 而言, 其同样具有近乎相 同的晶胞参数(表 S1). 其单晶结构中较为明显的区别在 于 MI-A 存在较多的 $\mathrm{C}=\mathrm{O} \cdots \mathrm{H}-\mathrm{C}$ 分子间相互作用 $(2.717,2.703 \AA)$ (图 S13), 使得晶体密度较 MI-B 和 MI-C 大. 这些结果表明分子构象和排列方式的微小变 化也会对发光特性产生巨大影响. 这些微小变化可能是 微量溶剂参与结晶造成的, 这一猜测也得到了核磁结果 的证实. 如图 S2 所示, 晶体溶解于氝代 DMSO $\left(\mathrm{DMSO}-d_{6}\right)$ 后，其 ${ }^{1} \mathrm{H}$ NMR 谱图中可明显观察到相应溶 剂峰. 形成晶体后, 分子构象在一定程度上被固定, 但 受微量溶剂作用，键长和键角的驰豫不同导致分子内与 分子间相互作用的微小改变. 在簇聚状态时, 簇生色团 的微环境因此改变, 使得其发光性质产生差异. (a)

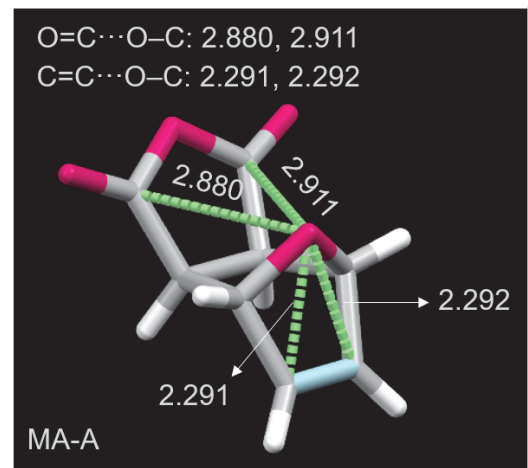

(b)

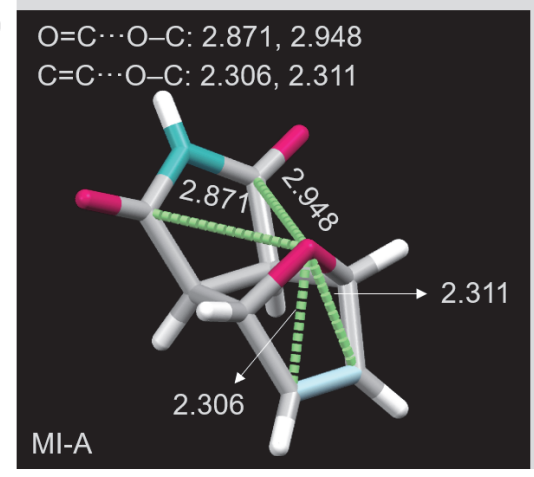

intramolecular interactions

(c)

\begin{tabular}{cccc}
\hline Sample & Solvent & $\begin{array}{c}\text { Space } \\
\text { group }\end{array}$ & $\begin{array}{c}\text { Density } \\
{\left[\mathrm{g} / \mathrm{cm}^{3}\right]}\end{array}$ \\
\hline MA-A & DCM & Orthorhombic & 1.541 \\
MA-B & EA & P2(1)2(1)2(1) & 1.540 \\
MA-C & DCM & & 1.541 \\
MI-A & EA & Monoclinic & 1.568 \\
MI-B & $\mathrm{NH}_{3} \cdot \mathrm{H}_{2} \mathrm{O}$ & P2(1)/c & 1.518 \\
MI-C & DCM & & 1.516 \\
\hline
\end{tabular}
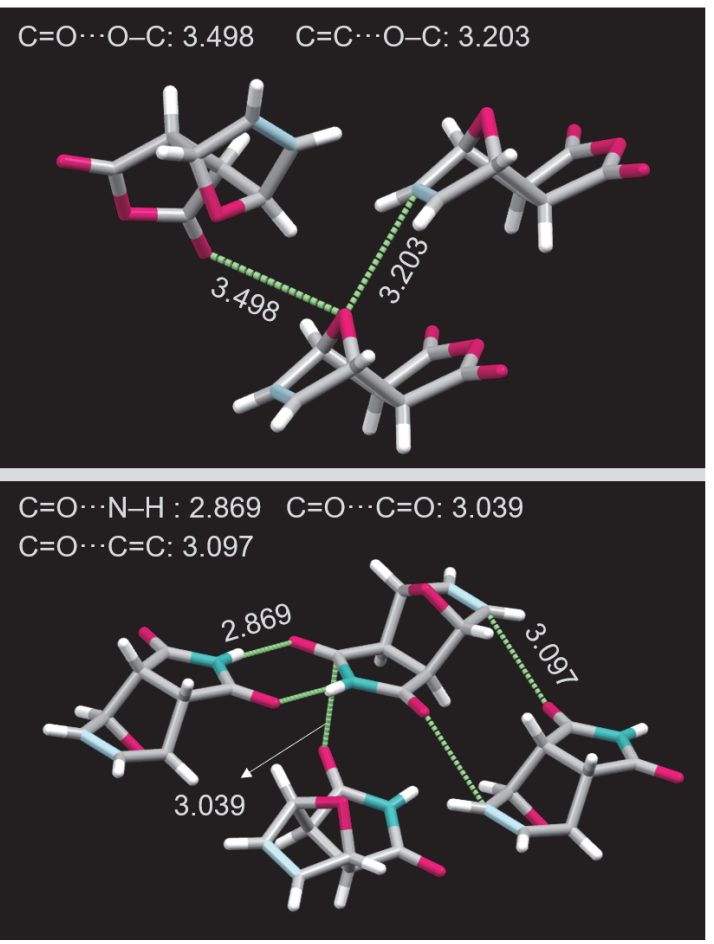

intermolecular interactions

(d)

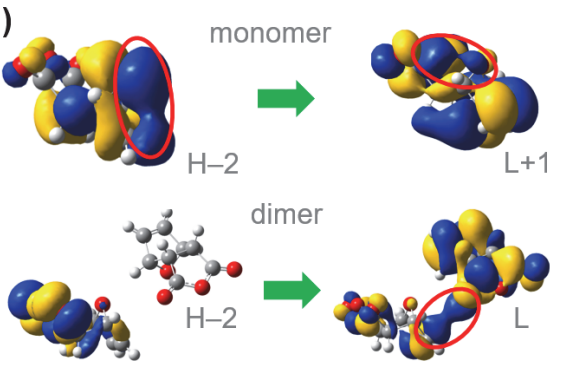

图 4 (a)MA-A 和(b)MI-A 单晶中分子的分子内作用力和排列堆积; (c)F-MA 和 F-MI 的单晶数据; (d)F-MA 单分子和二聚体在不同能级的电子云 密度分布

Figure 4 Crystal structures $(298 \mathrm{~K}$ ) with denoted intramolecular interactions and molecular packing with highlighted through-space conjugation for (a) MA-A and (b) MI-A. (c) Single crystal data of crystals of F-MA and F-MI. (d) Electron density distributions of different orbital levels of monomer and dimer of F-MA 
F-MI 分子的辐射跃迁的速率常数 $\left(k_{\mathrm{r}}^{\mathrm{p}}\right)$ 和非辐射跃 迁速率常数 $\left(k_{\mathrm{nr}}^{\mathrm{p}}\right)$ 较 F-MA 均显著减小. 这是由于 $\mathrm{N}$ 原子 的引入促进了体系氢键的形成, 因此其具有更紧密的分 子堆积和刚性环境 ${ }^{[25]}$, 使得分子的振动、转动等非辐射 跃迁被限制, 表现出 p-RTP 发射, 且 $\Phi_{\mathrm{c}}$ 和 $\Phi_{\mathrm{p}}$ 较 F-MA 显著提高(表 S2). 为了进一步阐释 F-MA 和 F-MI 的发 光现象, 我们对 F-MA 和 F-MI 分子进行了理论计算(图 $4 \mathrm{~d}, \mathrm{~S} 14)$. 由电子云分布图可以看出, F-MA 分子内存在 明显的 $\mathrm{C}=\mathrm{O} \cdots \mathrm{O}-\mathrm{C}$ 及 $\mathrm{C}=\mathrm{C} \cdots \mathrm{O}-\mathrm{C}$ 间的电子离域. 由 单分子到三聚体, 电子离域扩程度增加, 激发能总体下 降(图 S14, 表 S3, S4), 从而有利于激发. 在聚集过程中, 分子空间共轭程度进一步扩展, 形成簇聚种后, 可产生 共轭程度更大的聚集态, 从而易于激发产生可见光发 射.

\section{3 结论}

依据 CTE 机理, 我们研究了 F-MA 和 F-MI 两种具 有显著分子内与分子间空间共轭结构的非典型发光化 合物. 其结构中 $\mathrm{C}=\mathrm{O}$ 和杂原子的存在可有效促进系间 窝越(ISC)过程, 同时簇聚产生空间共轭使能隙降低, 也 有利于 ISC 发生, 从而赋予两种化合物荧光-磷光双发 射能力. 在不同条件获得的同质多晶, 由于微量溶剂分 子的存在, 使晶体构象产生微小差异, 分子簇聚状态所 处微环境改变, 进而改变了单线态和三线态的发射比 例，导致各同质多晶体的发光效率与颜色呈现差异. 与 F-MA 相比, F-MI 中氢键的引入使其 RTP 量子效率和寿 命均显著提高. 对于非典型发光化合物, F-MA 与 F-MI 的 RTP 及同质多晶现象揭示了分子结构与发射间的密 切关系, 有力地支持了 CTE 机理, 从而为非芳香性发光 化合物的分子设计和机理研究提供了新的启示.

\section{4 实验部分}

\subsection{F-MA 单晶的培养}

称取 $1.00 \mathrm{~g}$ F-MA(Aladdin)固体于 $50 \mathrm{~mL}$ 双颈烧瓶 中, 加入 $25 \mathrm{~mL}$ 二氯甲烷, 加热至 $50{ }^{\circ} \mathrm{C}$ 使其完全溶解, 待自然冷却后得到 F-MA 晶体, 命名为 MA-A, 抽滤得 到析出的晶体, 并在 $45{ }^{\circ} \mathrm{C}$ 真空烘箱中干燥过夜. ${ }^{1} \mathrm{H}$ NMR (500 MHz, DMSO- $\left.d_{6}\right) \delta: 6.59$ (d, $\left.J=1.0 \mathrm{~Hz}, 2 \mathrm{H}\right)$, 5.35 (t, $J=0.9 \mathrm{~Hz}, 2 \mathrm{H}), 3.32$ (s, 2H); ${ }^{13} \mathrm{C}$ NMR (126 MHz, $\mathrm{DMSO}-d_{6}$ ) $\delta: 172.01,137.33,82.11,49.56$ (图 S1). 分别 取出 $50 \mathrm{mg}$ 晶体溶解于乙酸乙酯和二氯甲烷形成饱和溶 液, 在室温下缓慢挥发分别获得 MA-B 和 MA-C 单晶.

\subsection{F-MI 的合成及单晶培养}

如图 1a 所示, 在带有冷凝器的 $50 \mathrm{~mL}$ 三口烧瓶中 加入 $3.00 \mathrm{~g}$ F-MA 固体、 $25 \mathrm{~mL}$ 氨水和 $5 \mathrm{~mL}$ 去离子水, 在室温下搅拌得到无色透明溶液. 将溶液加热回流 $4 \mathrm{~h}$ 后缓慢冷却至室温, 冷却过程中晶体逐渐析出, 抽滤得
到晶体. 将其在 $45{ }^{\circ} \mathrm{C}$ 真空烘箱中干燥过夜后得到 2.39 g F-MI 晶体, 产率 $80.1 \%$, 命名为 MI-B. ${ }^{1} \mathrm{H}$ NMR (500 MHz, DMSO- $\left.d_{6}\right) \delta: 11.13(\mathrm{~s}, 1 \mathrm{H}), 6.54(\mathrm{~d}, J=0.9 \mathrm{~Hz}, 2 \mathrm{H})$, $5.12(\mathrm{t}, J=1.0 \mathrm{~Hz}, 2 \mathrm{H}), 2.85(\mathrm{~s}, 2 \mathrm{H}) .{ }^{13} \mathrm{C}$ NMR $(126 \mathrm{MHz}$, DMSO- $d_{6}$ ) $\delta: 178.33,136.96,80.79,48.91$ (图 S2). 取 300 $\mathrm{mg}$ 晶体溶解于少量乙酸乙酯和二氯甲烷形成饱和溶液, 在室温下缓慢挥发得到单晶 MI-A 和 MI-C.

\section{3 理论计算}

由 F-MA 和 F-MI 单晶数据中分别选取单分子(单 体)、二聚体、三聚体和四聚体，使用含时密度泛函理论 (TD-DFT)在 Gaussian16 程序(版本 A.03)上进行计算. 通 过使用 B3LYP 混合函数和 $6-31+\mathrm{g}(\mathrm{d}, \mathrm{p})$ 基组计算得到第 $n$ 单线态 $\left(\mathrm{S}_{\mathrm{n}}\right)$ 和第 $n$ 三线态 $\left(\mathrm{T}_{\mathrm{n}}\right)$ 激发能及跃迁特性 ${ }^{[26]}$.

\section{致谢}

感谢上海交通大学仪器分析中心王瑞斌老师对光 谱测试的支持.

\section{References}

[1] (a) Wang, Z.; Cai, J.; Zhang, M.; Zheng, C.; Ji, B. Acta Chim. Sinica 2019, 77, 263 (in Chinese). (王志强, 蔡佳林, 张明, 郑才俊, 吉保 明, 化学学报, 2019, 77, 263.); (b) Zhou, J.; Liu, L.; Zhong, C.; Fu, Y.; Song, Z.; Peng, Y. Chin. J. Org. Chem. 2019, 39, 1444 (in Chinese). (周佳, 刘璐, 钟成, 傅杨, 宋智涁, 彭以元, 有机化学, 2019, 39, 1444.); (c) Chu, Y.; Xie, Z.; Zhuang, D.; Yue, Y.; Yue, Y.; Shi, W.; Feng S. Chin. J. Chem. 2019, 37, 1216.

[2] (a) Gong, Y.; Tan, Y.; Mei, J.; Zhang, Y.; Yuan, W. Z.; Zhang, Y.; Sun, J.; Tang, B. Z. Sci. Chin. Chem. 2013, 56, 1178; (b) Chen, X.; Wang, Y.; Zhang, Y.; Yuan, W. Z. Prog. Chem. 2019, 31, 1560 (in Chinese). (陈晓红, 王允中, 张永明, 袁望章, 化学进展, 2019, 31, 1560.); (c) Tomalia, D. A.; Klajnert M. B.; Johnson, K. A. M.; Brinkman, H. F.; Janaszewska, A.; Hedstrand, D. M. Prog. Polym Sci. 2019, 90, 35; (d) Zhang, H.; Zhao, Z.; McGonigal, P. R.; Ye, R.; Liu, S.; Lam, J. W. Y.; Kwok, R. T. K.; Yuan, W. Z.; Xie. J.; Rogach, A. L.; Tang, B. Z. Mater. Today 2020, 32, 275; (e) Tao, S.; Zhu, S.; Feng, T.; Zheng, C.; Yang, B. Angew. Chem., Int. Ed. 2020, 59, 9826.

[3] (a) Zhou, Q.; Yang, T.; Zhong, Z.; Kausar, F.; Wang, Z.; Zhang, Y.; Yuan, W. Z. Chem. Sci. 2020, 11, 2926; (b) Zheng, S.; Hu, T.; Bin, X.; Wang, Y.; Yi, Y.; Zhang, Y.; Yuan, W. Z. ChemPhysChem 2020, 21, 36; (c) Yan, J.; Zheng, B.; Pan, D.; Yang, R.; Xu, Y.; Wang, L.; Yang, M. Polym. Chem. 2015, 6, 6133; (d) Wang, D.; Wang, X.; Xu, C.; Ma, X. Sci. Chin. Chem. 2019, 62, 430; (e) Wang, Q.; Li, B.; Cao, H.; Jiang, X.; Kong, X. Z. Chem. Eng. J. 2020, 388, 124182; (f) Zhou, Q.; Wang, Z.; Dou, X.; Wang, Y.; Liu, S.; Zhang, Y.; Yuan, W. Z. Mater. Chem. Front. 2019, 3, 257.

[4] (a) Fang, M.; Yang, J.; Xiang, X.; Xie, Y.; Dong, Y.; Peng, Q.; Li, Q.; Li, Z. Mater. Chem. Front. 2018, 2, 2124; (b) Zheng, S.; Zhu, T.; Wang, Y.; Yang, T.; Yuan, W. Z. Angew. Chem., Int. Ed. 2020, 59, 10018; (c) Wang, Y.; Tang, S.; Wen, Y.; Zheng, S.; Yang, B.; Yuan, W. Z. Mater. Horiz. 2020, 7, 2105; (d) Yuan, L.; Yan, H.; Bai, L. Niu, S.; Du, Y.; Huang, W. Polym. Bull. 2018, 3, 24 (in Chinese). (原 璐瑶，颜红侠，白利华，牛松，杜玉群，黄为，高分子通报，2018, 3, 24.); (e) Yang, J. G.; Li, Y.; Wang, X. A.; Wang, D.; Sun, Y. W.; Wang, J. Q.; Xu, H. Acta Chim. Sinica 2019, 77, 1279 (in Chinese). (杨靖鸽, 李阳, 王小艾, 王栋, 孙亚伟, 王继乾, 徐海, 化学学 报, 2019, 77, 1279).

[5] Wang, D.; Imae, T. J. Am. Chem. Soc. 2004, 126, 13204.

[6] Zhou, Q.; Cao, B.; Zhu, C.; Xu, S.; Gong, Y.; Yuan, W. Z.; Zhang, Y. Small 2016, 12, 6586.

[7] Chen, X.; Luo, W.; Ma, H.; Peng, Q.; Yuan, W. Z.; Zhang, Y. Sci. China Chem. 2018, 61, 351 .

[8] Wang, Q.; Dou, X.; Chen, X.; Zhao, Z.; Wang, S.; Wang, Y.; Sui, K.; 
Tan, Y.; Gong, Y.; Zhang, Y.; Yuan, W. Z. Angew. Chem., Int. Ed. 2019, 58, 12667.

[9] (a) Bai, L.; Yan, H.; Bai, T.; Guo, L.; Lu, T.; Zhao, Y.; Li, C. Biomacromolecules 2020, 21, 3274; (b) Wang, S.; Wu, D.; Yang, S.; Lin, Z.; Ling, Q. Mater. Chem. Front. 2020, 4, 1198.

[10] (a) Lee, W. I.; Bae, Y.; Bard. A. J. J. Am. Chem. Soc. 2004, 126, 8358; (b) Wang, D.; Imae, T.; Miki. M. J. Colloid Interface Sci. 2007, 306, 222.

[11] Lu, H.; Feng, L.; Li, S.; Zhang, J.; Lu, H.; Feng, S. Macromolecules 2015, $48,476$.

[12] Shukla, A.; Mukherjee, S.; Sharma, S.; Agrawal, V.; Kishan, K. V. R.; Guptasarma, P. Arch. Biochem. Biophys. 2004, 428, 144.

[13] Pinotsi, D.; Grisanti, L.; Mahou, P.; Gebauer, R.; Kaminski, C. F.; Hassanali, A.; Schierle, G. S. K. J. Am. Chem. Soc. 2016, 138, 3046.

[14] Zhu, S.; Song, Y.; Shao, J.; Zhao, X.; Yang, B. Angew. Chem., Int. Ed. 2015, 54, 14626.

[15] (a) He, Z.; Li, W.; Chen, G.; Zhang, Y.; Yuan, W. Z. Chin. Chem. Lett. 2019, 30, 135; (b) Wang, K.; Xie, Y.; Liu, M.; Tao, W.; Zhang, H.; Huang, M.; You, J.; Liu, Y.; Li, Y.; Li, Z.; Dong, Y. Q. Adv. Optical Mater. 2020, 8, 2000436; (c) Liu, X.; Jia, Y.; Jiang, H.; Gao G.; Xia M. Acta Chim. Sinica 2019, 77, 1194 (in Chinese). (刘笑静, 贾彦荣, 江豪, 高贯雷, 夏敏, 化学学报, 2019, 77, 1194); (d) Huang, G.; Jiang, Y.; Yang, S.; Li, B. S.; Tang, B. Z. Adv. Funct. Mater. 2019, 29, 1900516; (e) Zhu, Ji.; Li, C.; Chen, P.; Ma, Z.; Zou, B.; Niu, L.; Cui, G.; Yang Q. Mater. Chem. Front. 2020, 4, 176.

[16] (a) Zhang, G.; Lu, J.; Sabat, M.; Fraser, C. L. J. Am. Chem. Soc. 2010, 132, 2160; (b) Yang, J.; Ren, Z.; Chen, B.; Fang, M.; Zhao, Z.; Tang, B. Z.; Peng, Q.; Li, Z. J. Mater. Chem. C 2017, 5, 9242; (c) Yan, D.; Evans, D. G. Mater. Horiz. 2014, 1, 46; (d) Wang, K.; Zhang, H.; Chen, S.; Yang, G.; Zhang, J.; Tian, W.; Su, Z.; Wang, Y. Adv. Mater. 2014, 26, 6168; (e) Li, W.; Huang, Q.; Mao, Z.; Zhao, J.; Wu, H.; Chen, J.; Yang, Z.; Li, Y.; Yang, Z.; Zhang, Y.; Aldred, M. P.; Chi, Z. Angew. Chem., Int. Ed. 2020, 59, 3739; (f) Liu, M. L.; Wu, Q.; Shi, H. F.; An, Z. F.; Huang, W. Acta Chim. Sinica 2018, 76, 246 (in Chinese). (刘明丽, 吴琪, 史慧芳, 安众福, 黄维, 化学学 报, 2018, 76, 246).

[17] (a) Yang, J.; Zhen, X.; Wang, B.; Gao, X.; Ren, Z.; Wang, J.; Xie, Y.; Li, J.; Peng, Q.; Pu, K.; Li, Z. Nat. Commun. 2018, 9, 840; (b) Wu, H.; Chi, W.; Baryshnikov, G.; Wu, B.; Gong, Y.; Zheng, D.; Li, X.; Zhao, Y.; Liu, X.; Ågren, H.; Zhu, L. Angew. Chem., Int. Ed. 2019, 58, 4328; (c) Lu, B.; Liu, S.; Yan, D. Chin. Chem. Lett. 2019, 30, 1908.

[18] Wang, Y.; Zhao, Z.; Yuan, W. Z. ChemPlusChem 2020, 85, 1065.

[19] (a) Yuan, L.; Yan, H.; Bai, L.; Bai, T.; Zhao, Yan.; Wang, L.; Feng, Y. Macromol. Rapid Commun. 2019, 40, 1800658; (b) Feng, Y.; Yan, H.; Ding, F.; Bai, T.; Nie, Y.; Zhao, Y.; Feng, W.; Tang, B. Z. Mater. Chem. Front. 2020, 4, 1375.
[20] (a) Shang, C.; Zhao, Y.; Wei, N.; Zhuo, H.; Shao, Y.; Wang H. Macromol. Chem. Phys. 2019, 220, 1900324; (b) Liu, B.; Zhang, H.; Liu, S.; Sun, J.; Zhang, X.; Tang, B. Z. Mater. Horiz. 2020, 7, 987.

[21] (a) Yuan, W. Z.; Shen, X. Y.; Zhao, H.; Lam, J. W. Y.; Tang, L.; Lu, P.; Wang, C.; Liu, Y.; Wang, Z.; Zheng, Q. J. Phys. Chem. C 2010 114, 6090; (b) Yang, J.; Chi, Z.; Zhu, W.; Tang, B. Z.; Li, Z. Sci. Chin. Chem. 2019, 62, 1090; (c) Xu, S.; Chen, R.; Zheng, C.; Huang, W. Adv. Mater. 2016, 28, 9920; (d) Gan, N.; Wang, X.; Ma, H.; Lv, A.; Wang, H.; Wang, Q.; Gu, M.; Cai, S.; Zhang, Y.; Fu, L.; Zhang, M.; Dong, C.; Yao, W.; Shi, H.; An, Z.; Huang, W. Angew. Chem., Int. Ed. 2019, 58,14140; (e) Zhang, L.; Li, M.; Gao, Q. Y.; Chen, C. F. Chin. J. Org. Chem. 2020, 40, 516 (in Chinese). (张亮, 李猛, 高庆宇, 陈传峰, 有机化学, 2020, 40, 516).

[22] CCDC numbers of the crystals: MA-A(2022799), MA-B(2022804), MA-C(2022803), $\quad$ MI-A(2022802), $\quad$ MI-B(2022801) MI-C(2022800).

[23] (a) Shang, C.; Zhao, Y.; Long, J.; Ji, Y.; Wang, H. J. Mater. Chem. C 2020, 8, 1017; (b) Wang, J.; Huang, Z. Z.; Ma, X.; Tian, H. Angew. Chem., Int. Ed. 2020, 59, 9928; (c) Shang, C.; Wei, N.; Zhuo, H.; Shao, Y.; Zhang, Q.; Zhang, Z.; Wang, H. J. Mater. Chem. C 2017, 5, 8082; (d) Yang, Z.; Ubba, E.; Huang, Q.; Mao, Z.; Li, W.; Chen, J.; Zhao, J.; Zhang, Y.; Chi, Z. J. Mater. Chem. C 2020, 8, 7384.

[24] Liu, B.; Chu, B.; Wang, Y.; Chen, Z.; Zhang, X. Adv. Optical Mater. 2020, 1902176

[25] (a) Ma, X.; Xu, C.; Wang, J.; Tian, H. Angew. Chem., Int. Ed. 2018 , 57, 10854; (b) Yan Z. A.; Zou, L.; Ma, X. Chin. J. Org. Chem. 2020 40, 1814 (in Chinese). (严子昂, 邹雷, 马骧, 有机化学, 2020, 40, 1814); (c) Zhang, T.; Ma, X.; Wu, H. W.; Zhu, L. L.; Zhao, Y. L.; Tian, H. Angew. Chem., Int. Ed. 2020, 59, 11206.

[26] Gaussian 16, Revision A.03, M. J. Frisch, G. W. Trucks, H. B. Schlegel, G. E. Scuseria, M. A. Robb, J. R. Cheeseman, G. Scalmani, V. Barone, G. A. Petersson, H. Nakatsuji, X. Li, M. Caricato, A. V. Marenich, J. Bloino, B. G. Janesko, R. Gomperts, B. Mennucci, H. P. Hratchian, J. V. Ortiz, A. F. Izmaylov, J. L. Sonnenberg, D. Williams-Young, F. Ding, F. Lipparini, F. Egidi, J. Goings, B. Peng, A. Petrone, T. Henderson, D. Ranasinghe, V. G. Zakrzewski, J. Gao, N. Rega, G. Zheng, W. Liang, M. Hada, M. Ehara, K. Toyota, R. Fukuda, J. Hasegawa, M. Ishida, T. Nakajima, Y. Honda, O. Kitao, H. Nakai, T. Vreven, K. Throssell, J. A. Montgomery, Jr., J. E. Peralta, F. Ogliaro, M. J. Bearpark, J. J. Heyd, E. N. Brothers, K. N. Kudin, V. N. Staroverov, T. A. Keith, R. Kobayashi, J. Normand, K. Raghavachari, A. P. Rendell, J. C. Burant, S. S. Iyengar, J. Tomasi, M. Cossi, J. M. Millam, M. Klene, C. Adamo, R. Cammi, J. W. Ochterski, R. L. Martin, K. Morokuma, O. Farkas, J. B. Foresman, and D. J. Fox, Gaussian, Inc., Wallingford CT, 2016. 